\title{
Comportamiento de corrosión-erosión en recubrimientos de NbN depositados mediante sputtering magnetrón
}

\author{
Erosion-corrosion behavior of magnetron sputtered $\mathrm{NbN}$ films
}

\author{
A. Cáceres $^{1} \quad$ J.J. Olaya ${ }^{1} \quad$ J.E. Alfonso ${ }^{2}$
}

Recibido 2 de mayo de 2011, aceptado 27 de julio de 2012

Received: May 2, $2011 \quad$ Accepted: July 27, 2012

\begin{abstract}
RESUMEN
En la nueva generación de recubrimientos de uso industrial se incluye el nitruro de niobio $(\mathrm{NbN})$ debido a sus propiedades fisicoquímicas tales como: alta conductividad eléctrica, buenas propiedades mecánicas, alto punto de fusión e inercia química y además por ser un superconductor con temperatura crítica de $16 \mathrm{~K}$. En este trabajo se reporta el efecto del sinergismo corrosión y erosión sobre recubrimientos de $\mathrm{NbN}$ depositados sobre acero inoxidable 304 y silicio usando los sistemas de sputtering con magnetrón balanceado y desbalanceado. Los experimentos de erosión-corrosión se estudiaron en un sistema que varió la velocidad y el ángulo de impacto de las partículas de erosión. Para ello se utilizó un lodo de partículas de $\mathrm{SiO}_{2}$ en una solución de $0,5 \mathrm{M}$ de $\mathrm{H}_{2} \mathrm{SO}_{4}+3,5 \% \mathrm{NaCl}$ y una concentración de $30 \%$ en peso de partículas de $\mathrm{SiO}_{2}$. La microestructura, morfología y composición química de las películas fueron analizadas con difracción de rayos X (DRX), microscopia electrónica de barrido (MEB) y espectroscopia de fotoelectrones generados por rayos X (EFRX), respectivamente. Los resultados muestran que la resistencia a la erosión-corrosión de los recubiertos de $\mathrm{NbN}$ fue superior al sustrato y los mecanismos de desgaste sobre el recubrimiento dependen del ángulo de impacto y la velocidad de las partículas de erosión. Posibles razones de este comportamiento son discutidas en este trabajo.
\end{abstract}

Palabras clave: Corrosión, erosión, sputtering, nitruro de niobio, acero inoxidable.

\section{ABSTRACT}

New generation PVD coatings for industrial applications include the niobium nitride $(\mathrm{NbN})$ due to its excellent properties such as high chemical inertness, excellent mechanical properties, high electrical conductivity, high melting point, and a superconducting transition temperature around $16 \mathrm{~K}$. In this work we reported the results the study of corrosion and erosion effect on niobium nitride coatings deposited on AISI 304 stainless steel using unbalanced and balanced magnetron sputtering and then it were compared to the uncoated tool steel substrate. The corrosion-erosion experiments were studied in a test machine varied the different velocities and impact angles of the erodent particles. The slurry used consists of quartz particles suspended in a mixture of $0.5 \mathrm{M}$ sulphuric acid solution and $3.5 \% \mathrm{NaCl}$, whit cathodic protection. The film microstructure and chemical composition were analyzed by X-ray diffraction (XRD), cross-section scanning electron microscopy (SEM) and X-ray photoelectron spectroscopy (XPS). The results indicated that the erosion-corrosion resistance of the coated samples was found to be superior to that of the uncoated sample and the wearing mechanisms on the coating and the steel depending on the impact angle and the impact velocity of erodent particles. Possible reasons for such behavior are described in this paper.

Keywords: Corrosion, erosion, sputtering, niobium nitride, stainless steel.

1 Departamento de Ingeniería Mecánica y Mecatrónica. Universidad Nacional de Colombia. Bogotá, Colombia. E-mail: jjolaya@unal.edu.co

2 Departamento de Física, Grupo de Ciencia de Materiales y Superficies. Universidad Nacional de Colombia. Bogotá, Colombia. E-mail: jealfonsoo@unal.edu.co 


\section{INTRODUCCIÓN}

Una alternativa económica y competitiva ha sido la aplicación de recubrimientos duros mediante técnicas de deposición física en fase de vapor (PVD, por sus siglas en inglés), las cuales son empleadas ampliamente en la industria metalúrgica debido a que mejoran las propiedades tribológicas e incrementan la resistencia a la corrosión de los materiales metálicos que están sometidos a ambientes agresivos en atmósferas industriales. El ejemplo más típico son los recubrimientos de nitruro de titanio (TiN), producidos generalmente por técnicas de sputtering y arco catódico pulsado [1-2].

A pesar del incremento comercial de los recubrimientos duros y al gran volumen de literatura científica disponible, existe aún un pobre entendimiento de la relación entre las propiedades de las películas y su comportamiento en ambientes combinados de erosión-corrosión [2-3]. La unión de estos procesos genera un estado de sinergismo que generalmente acelera la degradación en las superficies. Actualmente, los trabajos científicos sobre el comportamiento de erosión-corrosión de recubrimientos duros son escasos y básicamente están orientados al estudio de películas en monocapas de TiN y CrN y multicapas de TiN/AlTiN y CrN/ $\mathrm{NbN}[2,4-9]$.

En esta investigación se propone participar con un estudio simultáneo del fenómeno corrosión-erosión sobre recubrimientos duros novedosos como el $\mathrm{NbN}$, el cual es un potencial candidato para su uso en una gran variedad de aplicaciones debido a su alta inactividad química, alto coeficiente de absorción térmica y buenas propiedades mecánicas. Inicialmente este recubrimiento fue estudiado en el área de superconductores (temperatura crítica 16 K) en los años 1960 [10]; además se encontraron otras propiedades interesantes como alta conductividad eléctrica y alto punto de fusión que lo hacen importante para uso en microelectrónica en la producción de barreras de difusión. En los últimos años su estudio se ha enfocado como recubrimiento sobre superficies metálicas para protegerlas contra el desgaste [11-12]. Por ejemplo, Rutherford y sus colaboradores [13] encontraron que los recubrimientos de $\mathrm{NbN}$ depositados sobre aceros rápidos usando el sistema de arco eléctrico aumentaban la resistencia al desgaste con respecto al recubrimiento de TiN. Además el $\mathrm{NbN}$ presenta una temperatura de oxidación que sobrepasa los $800^{\circ} \mathrm{C}$ a diferencia del TiN que se oxida a temperaturas en el rango de $250-300{ }^{\circ} \mathrm{C}[2,12]$.

En este trabajo, los recubrimientos se depositaron con el sistema de sputtering con magnetrón desbalanceado (UBM, por sus siglas en inglés) y balanceado (BM, por sus siglas en inglés). Estas técnicas son el resultado de continuos avances en los procesos PVD. La diferencia principal entre un magnetrón convencional y el desbalanceado es el grado de confinamiento magnético al cual se encuentra sometido el plasma. En el sistema convencional solo una pequeña región en frente del blanco contiene un plasma denso. Por lo tanto, solo esta parte recibe un alto bombardeo iónico. En el modo desbalanceado algunas de las líneas magnéticas se dirigen hacia el sustrato, permitiendo de esta forma que un número de electrones que se encuentran confinados en el blanco pueden seguir en forma de trayectorias helicoidales las líneas de campo magnético, en dirección al sustrato [12, 14]. Estos electrones arrastran iones del blanco hacia el sustrato por atracción coulombiana, logrando así una mayor densidad de corriente iónica sobre el sustrato, lo cual ha demostrado ser eficiente para modificar la microestructura de las películas y en particular para formar depósitos con mayor densidad y menor rugosidad [14]. Además, esta tecnología se encuentra en un continuo desarrollo debido a que las necesidades industriales reclaman películas con propiedades específicas.

Además de crecer las películas, en este trabajo se realiza un estudio comparativo sobre la evaluación del comportamiento sinérgico en erosión-corrosión de dos grupos de recubrimientos de $\mathrm{NbN}$ depositados sobre acero inoxidable austenítico AISI 304. Un grupo de películas fue depositado con el sistema de sputtering rf con magnetrón balanceado y un segundo grupo usando el sistema de sputtering d.c con magnetrón desbalanceado. En las películas obtenidas se estudió la microestructura con difracción de rayos X (DRX). La morfología y la composición química de las películas producidas fueron analizadas mediante microscopia electrónica de barrido (MEB) y espectroscopia de fotoelectrones generados por rayos $\mathrm{X}$ (EFRX), respectivamente. 


\section{DESARROLLO EXPERIMENTAL}

Los recubrimientos de $\mathrm{NbN}$ se obtuvieron con las técnicas de sputtering con una configuración magnética balanceada y otra desbalanceada. En el primer grupo, las películas producidas con el sistema de magnetrón convencional (muestra NbN-BM) se utilizó un equipo Alcatel modelo HS 2000. En este sistema se evacuó el oxígeno dentro de la cámara hasta una presión de $5 \times 10^{-6}$ mbar utilizando una bomba turbomolecular auxiliada con una bomba mecánica. Para producir el plasma se utilizó un blanco de $\delta$-NbN $(99,99 \%)$ en una atmósfera de nitrógeno y argón con presión de trabajo de $7 \times 10^{-3}$ mbar, con flujos de $\mathrm{N}_{2}$ de $2 \mathrm{sccm}$ y $\mathrm{Ar}$ de $20 \mathrm{scmm}$. La descarga se estableció con una potencia de $350 \mathrm{~W}$ mediante una fuente de radiofrecuencia $(13,6 \mathrm{MHz})$ y empleado una distancia de trabajo blanco sustrato, $d_{b-s}$, de $5 \mathrm{~cm}$, una temperatura del sustrato de $240{ }^{\circ} \mathrm{C}$ y un tiempo de proceso de 90 minutos para producir un espesor medio de $1,9 \mu \mathrm{m}$. Por otro lado, en el segundo grupo de recubrimientos se utilizó el sistema de magnetrón desbalanceado (muestra NbN-UBM). Se utilizó un blanco de $\mathrm{Nb}(99,95 \%)$, una distancia blanco sustrato de $5 \mathrm{~cm}$ y empleando flujo de nitrógeno de $2 \mathrm{sccm}$ y argón de $14 \mathrm{sccm}$, con una presión de trabajo de $9 \times 10^{-3} \mathrm{mbar}$. Se empleó una potencia de descarga de $200 \mathrm{~W}$ mediante una fuente dc, la temperatura del sustrato se mantuvo en $380{ }^{\circ} \mathrm{C}$ y el tiempo de depósito empleado fue de 30 minutos para obtener un espesor medio de $1.8 \mu \mathrm{m}$. Para mejorar la adherencia del sistema se depositó una película de $\mathrm{Nb}$ de $\sim 100 \mathrm{~nm}$ de espesor entre el sustrato y el recubrimiento de $\mathrm{NbN}$. Las películas de $\mathrm{Nb}$ fueron depositadas usando las mismas condiciones de depósito de los recubrimientos de $\mathrm{NbN}$, pero sin introducir $\mathrm{N}_{2}$ en la cámara.

Para establecer el comportamiento de los recubrimientos ante los ensayos de corrosión y erosión se efectuaron pruebas sobre acero AISI 304 y los recubrimientos NbN-UBM y NbN-BM. Para evaluar el fenómeno corrosivo se realizaron curvas de polarización potenciodinámica con una velocidad de barrido de $20 \mathrm{mV}^{*} \mathrm{~min}^{-1}$, obtenidas con el potenciostato BAS Zahner y un software Thales. La celda está compuesta por un electrodo de referencia de $\mathrm{Ag} / \mathrm{AgCl}$, un electrodo auxiliar de platino y el electrodo de trabajo. La evaluación del fenómeno erosivo-corrosión se realizó sumergiendo las muestras en un lodo compuesto por $\mathrm{H}_{2} \mathrm{SO}_{4}$ $0,5 \mathrm{M}+3,5 \% \mathrm{NaCl}$ y $30 \%$ de partículas de $\mathrm{SiO}_{2}$ malla 50/70 AFS, usando velocidades de impacto de $1.000,2.000$ y $3.400 \mathrm{rpm}$ y ángulos de impacto de $30^{\circ}, 60^{\circ}$ y $90^{\circ}$. Detalles del equipo de erosióncorrosión se encuentran en el trabajo de D. López y sus colaboradores [2].

Con el fin de evaluar el estado de las superficies antes y después de la exposición a los experimentos de erosión-corrosión, se estudió la morfología de los recubrimientos mediante MEB, utilizando electrones secundarios y retrodispersados. Para caracterizar la morfología transversal se depositaron películas de $\mathrm{NbN}$ sobre obleas de silicio y posteriormente fueron fracturadas. En los estudios se utilizaron un microscopio electrónico de barrido modelo JEOL 5910LV y un MEB modelo Hitachi S-4700 (emisión de campo); en los recubrimientos NbN-UBM y NbN-BM también se estudió la estructura DRX para determinar las fases cristalográficas presentes y la orientación preferencial de las mismas. El equipo utilizado fue un difractómetro tipo PW1710 con la línea $\mathrm{K} \alpha$ del cobre. Las mediciones se realizaron con una intensidad de corriente de $30 \mathrm{~mA}$, una diferencia de potencial de $40 \mathrm{kV}$ y con un barrido de $30^{\circ}$ a $90^{\circ}(2 \theta)$, configurado con un tiempo por paso de $0,50 \mathrm{~s}$ y un tamaño de paso de $0,020^{\circ}$ (20) en modo continuo, usando la configuración geométrica Bragg-Brentano.

La composición química se determinó mediante EFRX. Estas mediciones se realizaron con presiones menores a $3 \times 10^{-9}$ mbar, usando una resolución espacial de $500 \mu \mathrm{m}$ y una energía de paso de 50 y $20 \mathrm{eV}$ en los espectros completos y de alta resolución, respectivamente. Todos los valores de energía fueron corregidos por efectos de carga con referencia al pico 1s del oxígeno a $531 \mathrm{eV}$. Se realizó una limpieza con sputtering usando iones de $\mathrm{Ar}^{+}$para remover de la superficie los contaminantes, tales como exceso de carbono y oxígeno. Para las mediciones de EFRX en las muestras de NbN-BM se utilizó un espectrómetro Leybold-Heraeus LHS-10 usando radiación de AlK $\alpha$ y en las muestras NbN-UBM se empleó un sistema Termo-Scientific Multilab utilizando radiación $\mathrm{MgK} \alpha$. La dureza de los recubrimientos se realizó con microdureza Vicker, con carga de 25 gr. El espesor de los recubrimientos fue medido con un perfilómetro Dektak IIA, haciendo un barrido en una longitud de $500 \mu \mathrm{m}$. 


\section{RESULTADOS}

Los estados químicos en los nitruros metálicos generalmente se caracterizan por corrimientos en las energías de enlace de $\mathrm{N}_{1 \mathrm{~s}}$ y el respectivo metal. En la Figura 1a-1c se observan los espectros de alta resolución de EFRX para las películas de NbN-BM. Se pueden apreciar en el espectro $\mathrm{Nb} 3 \mathrm{~d}$ (Figura 1a) tres componentes: la señal más intensa está centrada en una energía de ligadura de 206,5 eV que es característica del nivel $\mathrm{Nb} 3 \mathrm{~d}_{5 / 2}$ del $\mathrm{Nb}_{2} \mathrm{O}_{5}$, la segunda componente aparece a 203,6 eV y está asociada a la fase $\mathrm{NbN}_{\mathrm{x}}$, mientras que la tercera componente se encuentra a $204,8 \mathrm{eV}$ y corresponde a la presencia de un oxinitruro de niobio [9]. El espectro $\mathrm{N}$ 1s registrado de la película mencionada se presenta en la Figura $1 b$. El espectro consta de dos componentes. Una señal a 397,6 eV que corresponde a $\mathrm{NbN}_{\mathrm{x}}$ y una señal pequeña a $399,9 \mathrm{eV}$ que corresponde a oxinitruros de $\mathrm{Nb}$ [15]. Finalmente, en la Figura 1c se presenta el análisis del espectro $\mathrm{O} 1 \mathrm{~s}$, el cual consta de tres contribuciones: una primera centrada en $529,8 \mathrm{eV}$ que corresponde a enlaces $\mathrm{Nb}-\mathrm{O}$ en el $\mathrm{Nb}_{2} \mathrm{O}_{5}$ [16], una segunda a $531,6 \mathrm{eV}$ que corresponde a grupos $\mathrm{OH}$ y una tercera a $532,9 \mathrm{eV}$ que corresponde a $\mathrm{H}_{2} \mathrm{O}$ adsorbida sobre la superficie. Estos resultados indican que las últimas capas de la película depositada (que son los accesibles mediante EFRX) tienen una composición compleja. El $\mathrm{Nb}_{2} \mathrm{O}_{5}$ es la fase más externa y debe formarse por la oxidación del $\mathrm{NbN}$, tras la exposición de la película a la atmósfera del laboratorio. El oxinitruro de $\mathrm{Nb}$ corresponde a una fase localizada entre el óxido de niobio más externo y el cuerpo de $\mathrm{NbN}$ de la película total [17].

Por otro lado, en la Figura 1d se muestra en detalle el espectro de alta resolución de la señal $\mathrm{Nb} 3 \mathrm{~d}$ del sistema NbN-UBM. Los valores de energías de enlace $\mathrm{Nb} 3 \mathrm{~d}_{5 / 2}$ y $\mathrm{N}_{1 \mathrm{~s}}$ fueron de $203,9 \mathrm{eV}$ y $397,7 \mathrm{eV}$, respectivamente. Estos resultados están de acuerdo con los reportados en la literatura $\left(\mathrm{Nb} \mathrm{3d_{5/2 }}=204 \mathrm{eV}\right.$ y $\mathrm{N}_{1 \mathrm{~s}}=398 \mathrm{eV}$ ) para la fase cúbica FCC del $\mathrm{NbN}$ $[12,19]$. Los espectros de alta resolución que el pico de $\mathrm{N}_{1 \mathrm{~s}}$ (no mostrados) se caracterizaron por estar bien definidos, ajustados a una forma gaussiana y con un ancho a la mitad del pico de aproximadamente $1,7 \mathrm{eV}$, que son coherentes con los publicados en otros trabajos [12, 20-21].

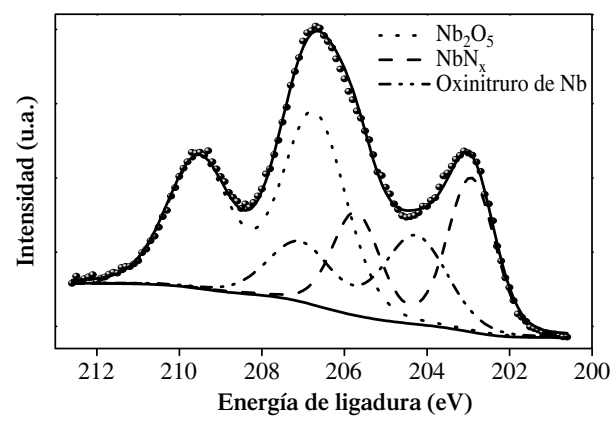

(a)

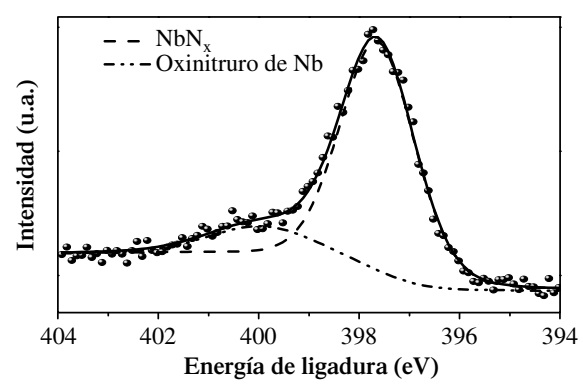

(b)

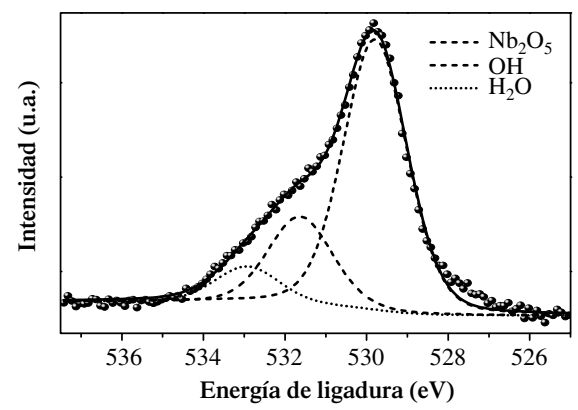

(c)

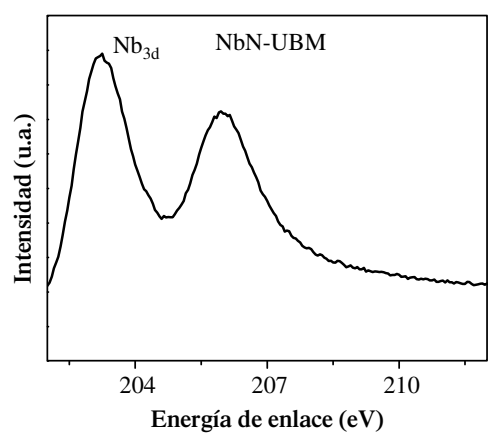

(d)

Figura 1. Espectros de XPS registrados de una película de $\delta-\mathrm{NbN}$ crecida: a) espectro $\mathrm{Nb} 3 \mathrm{~d}, \mathrm{~b}$ ) espectro N1s, c) espectro O1s, d). 
La Figura 2 muestra los patrones de DRX para los dos conjuntos de películas crecidas sobre acero AISI 304. Estos patrones están compuestos por planos bien definidos de la fase FCC del compuesto $\delta$-NbN (JCPDS $\mathrm{N}^{\circ} 38$-1135) y algunos casos aparecen los planos del sustrato y la fase BCC de $\mathrm{Nb}$, que corresponde a una intercapa de $\sim 100 \mathrm{~nm}$ de $\mathrm{Nb}$, que se depositó entre el sustrato y el $\mathrm{NbB}$ para mejorar la adherencia del sistema. Los picos de la muestra $\mathrm{NbN}-\mathrm{UBM}$ se caracterizan por ser más intensos y con una fuerte orientación preferencial a lo largo del plano (111) pero disminuye la intensidad relativa para la orientación (200). Por otro lado, la muestra $\mathrm{NbN}-\mathrm{BM}$ presenta una orientación mixta (111) y (200), ambos planos con baja intensidad relativa que sugiere una menor cristalinidad con respecto a las películas crecidas mediante magnetrón desbalanceado.

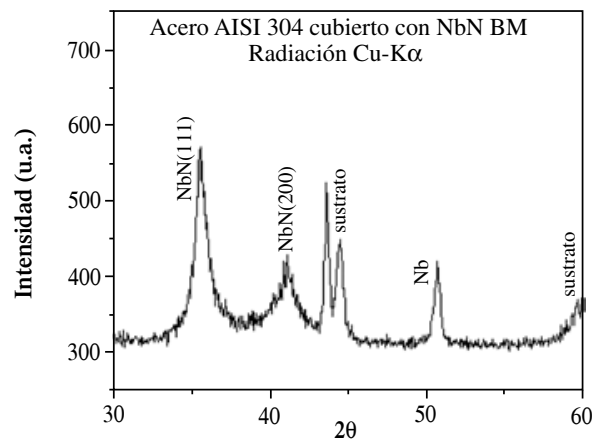

(a)

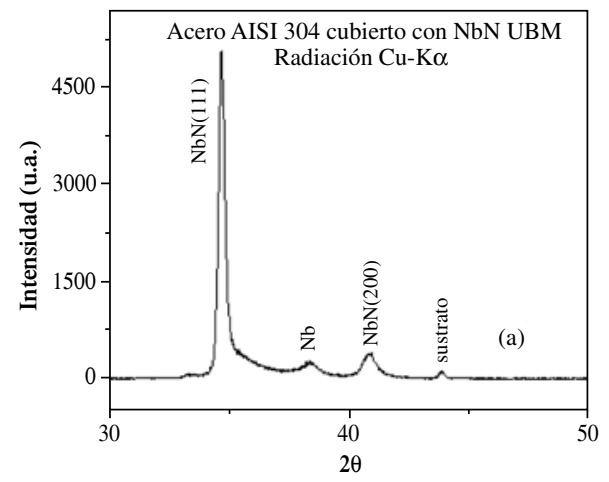

(b)

Figura 2. Espectro de difracción de rayos X: acero AISI 304 recubierto con $\mathrm{NbN}$ a) utilizando el sistema BM y b) utilizando el sistema UBM.
La Figura 3 muestra la sección transversal de los recubrimientos por microscopia electrónica de barrido, los cuales fueron crecidos sobre obleas de silicio y posteriormente fracturados para ser observados. Este método permitió estimar el espesor del recubrimiento; para el recubrimiento NbN-UBM se obtuvo un espesor medio de $1,8 \mu \mathrm{m}$ y para el recubrimiento de $\mathrm{NbN}-\mathrm{BM}$ un espesor medio de 1,9 $\mu \mathrm{m}$. En ambas morfologías se observa que los recubrimientos son compactos y densos, adicionalmente se evidencia microestructura columnar, correspondiente a la denominada zona $\mathrm{T}$ del modelo de estructura de zonas de Thorton [22].

La evaluación del fenómeno erosivo-corrosivo se realizó mediante mediciones de polarización potenciodinámica. En general, los resultados presentaron formas muy similares en las curvas de polarización; sin embargo, se observó un aumento de las densidades de corriente de corrosión con el incremento de la velocidad de impacto en la muestra NbN-BM (ver Figura 4). Por otro lado, en la muestra $\mathrm{NbN}-\mathrm{UBM}$ esta variación no fue significativa. También se puede apreciar que en estos experimentos no se logra definir la zona pasiva y no se aprecian cambios significativos en función del ángulo de impacto. Los resultados se resumen en

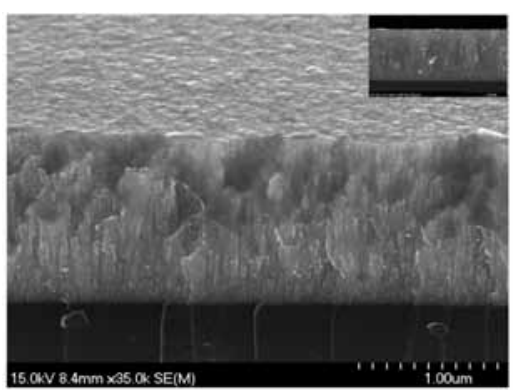

(a)

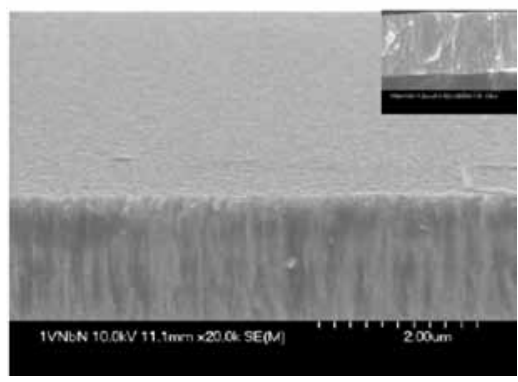

(b)

Figura 3. Sección transversal de los recubrimientos: (a) NbN-BM, (b) NbN-UBM. 
la Tabla 1, donde se observa que el recubrimiento NbN-BM impactado a $90^{\circ}$ y $1.000 \mathrm{rpm}$ exhibe una densidad de corriente de corrosión de un orden de magnitud menor con respecto al acero. Para los demás ángulos de impacto y revoluciones, los valores de $\mathrm{i}_{\text {corr }}$ no presentan un comportamiento sistemático, ya que en algunos casos es menor que el del acero sin recubrir y en otros es igual o ligeramente mayor.
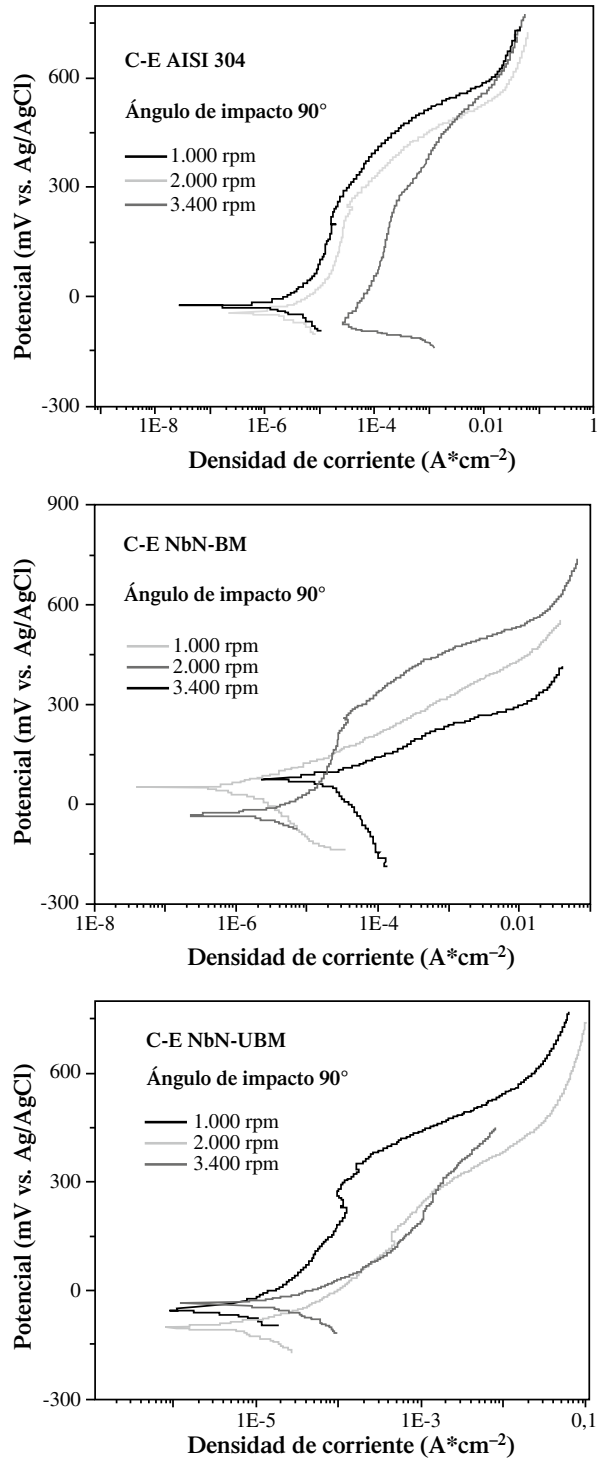

Figura 4. Curvas de polarización potenciodinámica obtenidas bajo condiciones de corrosiónerosión con un ángulo de impacto de 90 sobre (a) acero AISI 304 y los recubrimientos de $\mathrm{NbN}$ utilizando los sistemas (b) BM y (c) UBM.
Tabla 1. Resumen de los resultados de las curvas de polarización potenciodinámica para ensayos de corrosión-erosión de AISI 304, NbN-UBM y NbN-BM en solución $0,5 \mathrm{M} \mathrm{H}_{2} \mathrm{SO}_{4}+3,5 \% \mathrm{NaCl}$.

\begin{tabular}{|l|c|c|c|c|c|c|}
\hline & \multicolumn{2}{|c|}{$\mathbf{3 0}^{\circ}$} & \multicolumn{2}{c|}{$\mathbf{6 0}^{\circ}$} & \multicolumn{2}{c|}{$\mathbf{9 0}^{\circ}$} \\
\cline { 2 - 7 } & icorr* & Ecorr** $^{\circ}$ icorr* & Ecorr** & icorr* & Ecorr** \\
\hline & \multicolumn{7}{|c|}{$\mathbf{1 . 0 0 0}$ rpm } \\
\hline AISI 304 & $2.97 \mathrm{E}-6$ & -45 & $2.99 \mathrm{E}-6$ & -20 & $1.55 \mathrm{E}-6$ & -27 \\
\hline NbN-UBM & $2.29 \mathrm{E}-6$ & -109 & $2.01 \mathrm{E}-6$ & -44 & $1.06 \mathrm{E}-6$ & -52 \\
\hline NbN-BM & $2.99 \mathrm{E}-6$ & 34 & $3.01 \mathrm{E}-6$ & 38 & $9.49 \mathrm{E}-7$ & 42 \\
\hline & \multicolumn{7}{|c|}{$\mathbf{2 . 0 0 0}$ rpm } \\
\hline AISI 304 & $4.72 \mathrm{E}-6$ & -18 & $2.10 \mathrm{E}-6$ & -52 & $2.38 \mathrm{E}-6$ & -43 \\
\hline NbN-UBM & $4.86 \mathrm{E}-6$ & -105 & $1.15 \mathrm{E}-5$ & -39 & $1.69 \mathrm{E}-6$ & -105 \\
\hline NbN-BM & $1.29 \mathrm{E}-5$ & 38 & $1.67 \mathrm{E}-5$ & 42 & $2.69 \mathrm{E}-6$ & -32 \\
\hline & \multicolumn{7}{|c|}{3.400 rpm } \\
\hline AISI 304 & $4.82 \mathrm{E}-5$ & -26 & $1.74 \mathrm{E}-5$ & -61 & $3.36 \mathrm{E}-5$ & -75 \\
\hline NbN-UBM & $2.01 \mathrm{E}-5$ & -87 & $2.02 \mathrm{E}-5$ & -45 & $1.64 \mathrm{E}-5$ & -36 \\
\hline NbN-BM & $2.52 \mathrm{E}-5$ & 107 & $1.71 \mathrm{E}-5$ & 54 & $1.87 \mathrm{E}-5$ & 73 \\
\hline
\end{tabular}

* Densidad de corriente de corrosión en $\mathrm{A}^{*} \mathrm{~cm}^{-2}$

** Potencial de corrosión en $\mathrm{mV}$ vs. Ag/AgCl.

Con el fin de dar una mejor interpretación de los resultados se realizaron mediciones complementarias de pérdida de peso. En los ensayos de erosióncorrosión se presenta una pérdida significativa de peso con la variación del ángulo y la velocidad de impacto, tal como se observa en las Figuras 5a-c, donde se puede apreciar que los menores valores de desgaste se presentan en los sustratos de acero, lo que se puede atribuir al desarrollo de una gran deformación plástica generada por las partículas de erosión, ver Figura 6a.

El mecanismo de degradación del acero AISI 304 podría estar asociado al modelo para materiales dúctiles propuesto por Hutchings [23] en el cual la degradación del material se debe al aumento del número de ciclos de impacto de las partículas o al aumento de la velocidad de rotación. Esto involucra una acumulación de deformación plástica por el constante impacto de las partículas erosionantes y la generación de una placa superficial de material endurecido, el cual es removido totalmente cuando se alcanza un límite de deformación crítica o falla por fatiga. En este caso, la fuerza de impacto de las partículas no fue suficiente para generar un desprendimiento apreciable de material. 


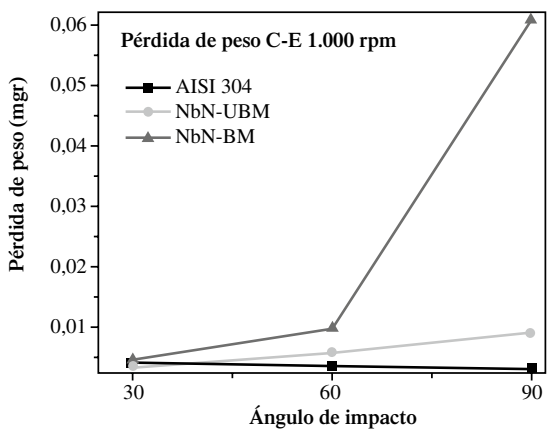

(a)

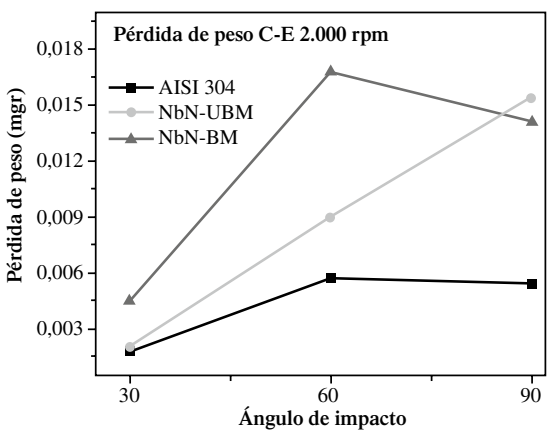

(b)

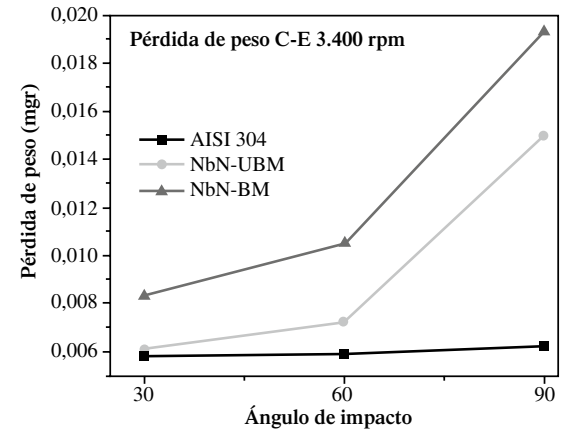

(c)

Figura 5. Pérdida de peso durante ensayo corrosión-erosión, velocidad de partículas erosionantes de a-1.000, b-2.000 y c-3.400 rpm.

En los recubrimientos se pudo observar mayor pérdida de peso a un ángulo de impacto de $90^{\circ}$, que representa la condición más severa para materiales de naturaleza frágil [2-4]. El anterior estudio también estableció que las películas crecidas con el sistema MB presentan mayores tasas de desgaste respecto a las crecidas con el sistema desbalanceado. Estos cambios pueden explicarse por la menor dureza que presentan estos recubrimientos. De acuerdo a los valores de dureza compuesta, los valores en películas de NbN-BM y NbN-UBM fueron de 1.200 y $2.300 \mathrm{~kg} / \mathrm{mm}^{2}$, respectivamente.

Por otro lado, el aumento de la dureza en el recubrimiento producido con el magnetrón desbalanceado redujo la pérdida de peso. El aumento de la dureza en esta configuración se puede atribuir a la formación de una estructura más compacta, que se favorece debido a que en el proceso de síntesis se logra una mayor ionización y disociación del plasma producido por la mayor temperatura electrónica, y un aumento de la densidad y energía de iones en la superficie de crecimiento [12]. Estos cambios aumentan la movilidad de los átomos adsorbidos que son los responsables en modificar los esfuerzos residuales, la densidad y la orientación preferencial.

$\mathrm{El}$ aumento de la dureza en el sistema NbN-UBM también puede ser atribuido a su orientación preferencial (111). Los planos (111) de un sistema FCC se caracterizan por tener un factor de Schmid cero [24], el cual representa la tendencia a iniciar deformación plástica para un sistema de deslizamiento determinado. Por lo tanto, la deformación plástica en un sistema cúbico FCC en la dirección (111) es menor, lo que implica mejorar la resistencia al desgaste erosivo-corrosivo.

Las Figuras 6 b-d presentan la superficie del sustrato y los recubrimientos después de los ensayos de erosión-corrosión. En la Figura $6 \mathrm{~b}$ se muestra un ejemplo del aspecto superficial en el sustrato sometido a ensayo de corrosión-erosión a $3.000 \mathrm{rpm}$ con un ángulo de recubrimiento impactado a $90^{\circ}$. La degradación en el sustrato puede estar asociada al componente químico, presentándose corrosión por picadura y corrosión intergranular. En este caso, se hace más notable la degradación superficial debida a fenómenos de corrosión, los cuales fueron acelerados por el componente mecánico de impacto de partículas erosionantes, las cuales promovieron la degradación de la superficie del material. El fenómeno de corrosión intergranular es muy común en aceros inoxidables austeníticos y se caracteriza por el ataque selectivo en la zona adyacente a los límites de grano del material. Generalmente ocurre por la precipitación de carburos de cromo en los límites de grano, reduciendo así el cromo en la matriz o regiones adyacentes a los límites de grano. 
(a)

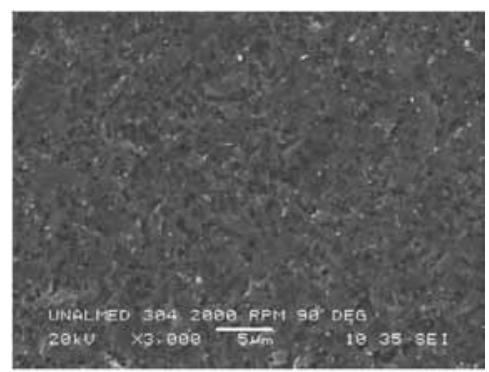

(b)

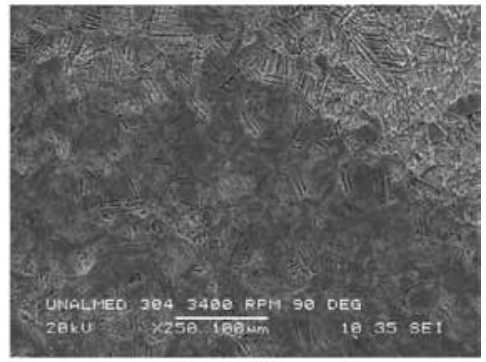

(c)

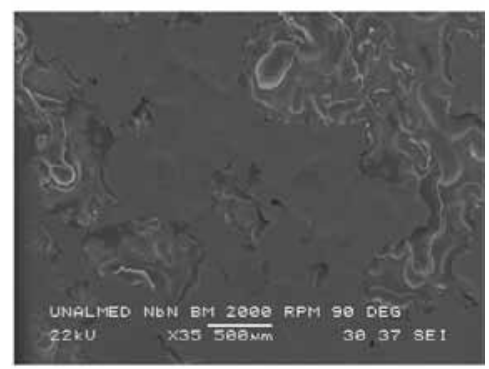

(d)

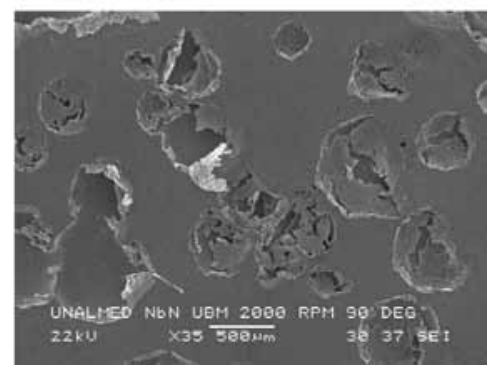

Figura 6. Aspecto superficial del sustrato: (a) después de un ensayo de erosión y (b) erosión-corrosión y los recubrimientos, (c) NbN-BM y (d) NbN-UBM sometidos a ensayo de corrosión-erosión con ángulo de impacto de $90^{\circ}$ y velocidad de partículas erosionantes $2.000 \mathrm{rpm}$.

Las Figuras 6 c-d muestran la superficie de los recubrimientos de NbN-UBM y NbN-BM en el ensayo de erosión-corrosión con $2.000 \mathrm{rpm}$ y un angulo de $90^{\circ}$. La superficie final del recubrimiento presentó degradación y/o delaminación del sustrato.
El grado de desgaste dependió de las diferentes condiciones de ensayos, es decir, de la naturaleza del material y el medio de simulación del experimento. La delaminación en algunas regiones del recubrimiento posiblemente es generada por la combinación de los siguientes tres fenómenos: (1) efecto mecánico generado por la acción repetida de impacto de las partículas a $90^{\circ}$ sobre la superficie, (2) la formación de celdas galvánicas y (3) los productos de corrosión que se generan en los defectos del recubrimiento y la interfase con el sustrato.

La degradación que se desarrolla en los recubrimientos producidos por sputtering puede explicarse por un mecanismo de corrosión en presencia de la solución acuosa con contenido de cloruros y desgaste por fatiga generado por los impactos repetitivos de las partículas de $\mathrm{SiO}_{2}$. En este mecanismo se debe tener en cuenta el grado de porosidad que tiene la microestructura columnar del recubrimiento (microporosidad), el cual se convierte en canal de difusión para que la solución corrosiva ataque la superficie del sustrato y formar un par galvánico. De esta forma se pueden producir productos de corrosión que se forman en la inter-fase y se genera pérdida de adherencia del recubrimiento, que además bajo la acción mecánica de partículas erosivas causarán el desprendimiento parcial de la película.

\section{CONCLUSIONES}

En este trabajo se caracterizó el comportamiento de erosión-corrosión de los recubrimientos de $\mathrm{NbN}$ depositados sobre el acero AISI 304 producidos por las técnicas de sputtering rf con magnetrón convencional y dc desbalanceado.

En general, los recubrimientos de $\mathrm{NbN}$ mejoraron la resistencia a los fenómenos de corrosión-erosión, esto debido principalmente a su naturaleza cerámica y sus mejores propiedades mecánicas. En las condiciones de ensayo de esta investigación, el recubrimiento de $\mathrm{NbN}$ producido con el sistema de sputtering desbalanceado presentó la menor degradación superficial. Esto puede ser explicado por el aumento de la dureza, una estructura más compacta y cristalina, con una orientación preferencial en la dirección (111).

Los recubrimientos de $\mathrm{NbN}$ presentaron un crecimiento microestructural columnar compacto; 
sin embargo, su microporosidad inherente generó un fenómeno de corrosión galvánica debido a que el recubrimiento es químicamente más noble que el acero. En condiciones simultáneas de corrosión-erosión se incrementó la degradación del recubrimiento debido seguramente por la formación de estas celdas galvánicas y, combinado bajo acción mecánica repetitiva de las partículas erosivas, se genera pérdida de adherencia y aceleración del desprendimiento parcial o localizado de la película.

\section{AGRADECIMIENTOS}

Los autores agradecen el soporte económico de Colciencias en el proyecto CT-206-2006, la DIB-UNAL y DGAPA-UNAM a través de los proyectos IN100203 y IN100701.

\section{REFERENCIAS}

[1] J. Wesley Cox. "Wear and Corrosion Resistant Hard Coatings for Non-Cutting Tool Applications". William Andrew Publishing/ Noyes, p. 411. New Jersey, U.S.A. 2001. ISBN: 9780815514381.

[2] D. López, C. Sánchez and A. Toro. "Corrosionerosion behavior of TiN-coated stainless steels in aqueous slurrie". Wear. Vol. 258, pp. 684-692. 2005.

[3] A. Cáceres and J.J. Olaya. "Erosión-corrosión de recubrimientos de $\mathrm{NbN}$ ". Tesis de maestría. Universidad Nacional de Colombia. Medellín, Colombia. 2008.

[4] Y.P. Purandare, A.P. Ehiasarian, M.M. Stack and P.Eh. Hovsepian. "CrN/NbN coatings deposited by HIPIMS: A preliminary study of erosion-corrosion performance". Surf. Coat. Technol. Vol 204, pp. 1158-1162. 2010.

[5] M.M. Stack, H.W. Wang and W.D. Münz. "Some thoughts on the construction of erosion-corrosion maps for PVD coated steels in aqueous environments". Surf. Coat. Technol. Vol. 113, pp. 52-62. 1999.

[6] M.M. Stack and H.W. Wang. "Simplifying the erosion-corrosion mechanism map for erosion of thin coatings in aqueous slurries". Wear. Vol. 233-235, pp. 542-551. 1999.

[7] Y. Purandare, M.M. Stack and P. Hovsepian. "A study of the erosion-corrosion of PVD $\mathrm{CrN} / \mathrm{NbN}$ superlattice coatings in aqueous slurries". Wear. Vol. 259, pp. 256-262. 2005.
[8] Y.P. Purandare, M.M. Stack and P.Eh. Hovsepian. "Velocity effects on erosioncorrosion of $\mathrm{CrN} / \mathrm{NbN}$ "superlattice" PVD coatings". Surf. Coat. Technol. Vol. 201, pp. 361-370. 2006.

[9] G. Jouve, C. Severac, S. Cantacuzene. "XPS study of $\mathrm{NbN}$ and (NbTi)N superconducting coatings". Thin Solid Films. Vol. 287, p. 994. 1996.

[10] J.J. Olaya, L. Huerta, S.E. Rodil and R. Escamilla. "Superconducting niobium nitride films deposited by unbalanced magnetron sputtering”. Thin Solid Films. Vol. 516, pp. 8768-8773. 2008.

[11] S.E. Rodil, J.J. Olaya, S. Muhl, B. Bhushan and G. Wei. "The influence of the magnetic field configuration on plasma parameters and microstructure of niobium nitride films". Surf. Coat. Technol. Vol. 201, pp. 6117-6121. 2007.

[12] J.J. Olaya, S.E. Rodil and S. Muhl, "Comparative study of niobium nitride coatings deposited by unbalanced and balanced magnetron sputtering". Thin Solid Films. Vol. 516, pp. 8319-8326. 2008.

[13] K.L. Rutherford, P.W. Hatto, C. Davies and I.M. Hutchings. "Abrasive wear resistance of $\mathrm{TiN} / \mathrm{NbN}$ multi-layers: measurement and neural network modelling". Surf. Coat. Technol. Vol. 86-87, pp. 472-479. 1996.

[14] J.J. Olaya, S.E. Rodil, S. Muhl and E. Sánchez. "Comparative study of chromium nitride coatings deposited by unbalanced and balanced magnetron sputtering". Thin Solid Films. Vol. 474, pp. 119-126. 2005.

[15] I. Bertoti. "Characterization of nitride coatings by XPS". Surf and coating Tech. Vol. 151, pp. 194-203. 2002.

[16] F.J. Garbassi. "XPS study of tellurium-niobium and tellurium-tantalum oxide systems". Electron Spectros. Relat. Phenon. Vol. 22, pp. 95-107. 1981.

[17] J.E. Alfonso, J. Buitrago, J. Torres, B. Santos and J.F. Marco. "Crystallographic structure and surface composition of $\mathrm{NbN}$ thin films grown by RF magnetron sputtering". Microelectronics Journal. Vol. 39, pp. 13271328. 2008.

[18] J.E. Alfonso, J. Buitrago, J. Torres, J.F. Marco and B. Santos. "Influence of fabrication parameters on crystallization, microstructure, 
and surface composition of $\mathrm{NbN}$ thin films deposited by rf magnetron sputtering". J. Mater. Sci. Vol. 45, pp. 5528-5533. 2010.

[19] G. Jouve, C. Séverac and S. Cantacuzène. "XPS study of $\mathrm{NbN}$ and (NbTi)N superconducting coatings". Thin Solid Films. Vol. 287, pp. 146-153. 1996.

[20] S.F. Ho, S. Contarini and J.W. Rabalais. "Ion-beam-induced chemical changes in the oxyanions (Moyn-) and oxides (Mox) where $\mathrm{M}=$ chromium, molybdenum, tungsten, vanadium, niobium and tantalum". J. Phys. Chem. Vol. 91, pp. 4779-4780. 1987.
[21] I. Bertóti. "Characterization of nitride coatings by XPS”. Surf. Coat. Technol. Vol. 151-152, pp. 194-203. 2002.

[22] Thornton. "The Microstructure of Sputterdeposited Coatings". J. Vac. Sci. Technol. A4. pp. 3059-3065. 1986.

[23] I.M. Hutchings. " 5 th International Conference on erosion by solid and liquid impact". University of Cambridge. 1979.

[24] H. Ljungcrntz, M. Odén, L. Hultman, J.E. Greene, J.-E. Sundgren. "Nanoindentation studies of single-crystal (001)-, (011)-, and (111)-oriented TiN layers on MgO”. J. Appl. Phys. Vol. 80, pp. 6725-6733. 1996. 